\title{
Flow and Heat Transfer in Turbulent Channel Flow over Circular Dimples: Effect of Location of the Deepest Point Of Dimples
}

\author{
B. C. Khoo' ${ }^{1}$ Y. Chen ${ }^{2}$, C. M. Tay ${ }^{1}$ \\ ${ }^{1}$ Dept. Mech. Eng., National University of Singapore \\ Kent Ridge, Singapore 119260 \\ ${ }^{2}$ Inst. High Performance Computing \\ 1 Fusionopolis Way, \#16-16 Connexis, Singapore 138632
}

\begin{abstract}
Circular axisymmetric dimples often exhibit a region of flow recirculation at the upstream portion of the dimple, leading to increased flow resistance and reducing heat transfer. To reduce this flow recirculation, the upstream wall slope may be reduced. Two methods of achieving this are to reduce the dimple depth, and to shift the deepest point of the dimple downstream. Circular nonaxisymmetric dimples with depth to diameter ratios up to $17.5 \%$ are studied numerically and experimentally in a fully developed turbulent channel regime. The results show that shallow dimples with depth to diameter ratios of 5\% and below are not favourable for heat transfer due to their weaker heat transfer enhancement ability. Deeper dimples with depth to diameter ratios of $10 \%$ and above exhibit significantly greater heat transfer enhancement. Movement of the deepest point of the dimple downstream have the effect of reducing flow recirculation at the upstream portion and increased fluid ejection at the downstream edge due to flow impingement there. Both these effects lead to improved heat transfer of the dimple surface. Movement of the deepest point of the dimple streamwise also affects the friction factor due to the dimples. For shallow dimples with depth to diameter ratios of 5\%, shifting the deepest point downstream reduces the friction factor due to the more significant effect the reduced flow separation has on the friction factor. For deeper dimples with depth to diameter ratios of $15 \%$, movement of the deepest point downstream however leads to increasing friction factor due to the more significant effect of the flow impingement on the friction factor for these deeper dimples.
\end{abstract}

Keywords: Channel Flow, Turbulent Flow, Dimple, Heat Transfer, Drag Reduction.

\section{Introduction}

Dimpled surfaces have attracted much attention due to its advantages over other methods to enhance heat transfer. They are relatively easy to manufacture, and can potentially increase heat transfer with a smaller penalty in pressure loss compared to other heat transfer enhancement devices [1 - 3]. The superior thermal performance of dimples is attributed to the strong ejections and secondary rotating motions observed near the downstream side of circular dimples [4-6].

Though deeper dimples (with depth $d$ to diameter $D$ ratios, $d / D>30 \%$ ) enjoy higher heat transfer rates, they also suffer from greater pressure losses, thereby restricting their applications. Hence instead of simply increasing the depth of axisymmetric circular dimples to increase heat transfer rates, new dimple shapes such as teardrop shaped dimples [7], dimples with streamwise ridges [8] and oval shaped dimples [9] have been investigated in efforts to obtain higher effective heat transfer efficiency of dimpled surfaces. Non-axisymmetric or non-circular dimples, potentially capable of both increasing heat transfer rates while suppressing pressure losses, may be more effective than axisymmetric ones in heat exchangers. However, such dimple shapes may be more difficult to manufacture compared with circular axisymmetric dimples [7,8] or may be limited to a smaller coverage area due to its specific geometry [9].

The pressure losses due to dimples are the combined result of skin friction and pressure drag [10]. Flow separation commonly observed at the upstream portion of the dimples cause significant pressure drag $[4,11]$, and any change in the dimple geometry to reduce this flow separation region results in a reduction in the pressure drag as well as the overall pressure losses due to the dimples. Reduction of the flow separation region where flow recirculation is present also often leads to increased heat transfer. Both these effects contribute to increase the heat transfer efficiency of the dimple surface. The easiest way to reduce the flow separation region at the upstream portion of the dimple is to reduce the wall slope at the upstream portion of the dimple [12]. Two ways of achieving this easily with a circular dimple is to reduce the dimple depth [11], or to shift the deepest point of the dimple downstream to create a non-axisymmetric circular dimple [1]. 
The motivation of the current study is to investigate these two methods to increase the heat transfer efficiency of dimpled surfaces by reducing the flow separation occurring at the upstream portion of circular axisymmetric dimples. Non axisymmetric dimples created by moving the deepest point of an axisymmetric circular dimple downstream along the dimple centreline are investigated in the current work. In this way, the plan view of the dimples remain circular, allowing efficient packing of the dimple depressions and achieving a high coverage ratio. The study involves multiple such dimples instead of a single dimple [9] arranged in the turbulent channel flow to investigate the performance of such dimples for heat transfer.

\section{Methodology}

The work uses both numerical simulations as well as experimental measurements to study circular non-axisymmetric dimples with depth to diameter ratios from 5\% to 15\%. Detached Eddy Simulation (DES) is used to study the dimples numerically. DES is a hybrid method that combines Reynolds-Averaged Navier-Stokes (RANS) for modelling the flow near the wall and Large Eddy Simulation (LES) for simulation of the flow higher up the wall and is suitable for such flows involving flow separation [13 - 15]. Details of the DES method used here may be found in Chen et al. [1]. Performance parameters such as the friction factor, Nusselt number, area and volume goodness factors [16] of the non-axisymmetric dimples in turbulent channel flow are investigated numerically using DES. The numerical simulation is carried out at Reynolds numbers $\operatorname{Re}_{2 h}$ (based on bulk velocity and full channel height $2 h$ ) ranging from 4,000 to 6,000 and Prandtl number $\operatorname{Pr}$ of 0.7 .

The non-axisymmetric dimples investigated in the present study is obtained by moving the deepest point streamwise by $\Delta x$ of an axisymmetric dimple with a cross-section as shown in Fig. 1. $D$ is the dimple diameter and $d$ is the dimple depth, and $\mathrm{R}$ and $r$ are varied to obtain different depth to diameter ratios.

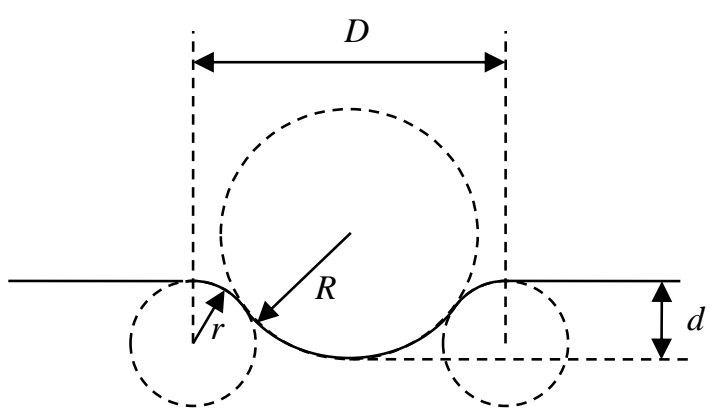

Fig. 1: Cross section of axisymmetric dimple.

The effect with different depth ratios and streamwise movement of the deepest point are investigated. The parameters of these dimples are given in

Table 1. The streamwise movement of the deepest point along the dimple centreline $\Delta x$ is normalised with the dimple diameter $D$ in the table, and negative values of $\Delta x / D$ means movement of the deepest point upstream from the dimple center, while positive values mean a downstream movement from the dimple center.

Table 1: Dimple parameters.

\begin{tabular}{|c|c|c|c|c|}
\hline Case & $D / h$ & $d / h$ & $d / D$ & $\Delta x / D$ \\
\hline 1 & 5 & 0.5 & $10 \%$ & -0.15 \\
\hline 2 & 5 & 0.5 & $10 \%$ & 0 \\
\hline 3 & 5 & 0.5 & $10 \%$ & 0.15 \\
\hline 4 & 5 & 0.5 & $10 \%$ & 0.175 \\
\hline 5 & 5 & 0.75 & $15 \%$ & -0.075 \\
\hline 6 & 5 & 0.75 & $15 \%$ & 0 \\
\hline 7 & 5 & 0.75 & $15 \%$ & 0.15 \\
\hline
\end{tabular}


Experiments have also been carried out on similar non-axisymmetric circular dimples with their deepest point shifted in the streamwise direction, though limited to shallow dimples with depth to diameter ratios of 5\% in a turbulent channel flow facility. Static pressure measurements have been made to evaluate the friction factor compared to that of the plane channel without dimples using the method of [17].

\section{Results and Discussion}

Fig. 2 shows the results for the friction factor $\mathrm{C}_{\mathrm{f}}$, Nusselt number $\mathrm{Nu}$, and the volume goodness ratio $\mathrm{Gv}$ as defined by Shah and London [16], as the depth to diameter ratio $(d / D)$ of circular axisymmetric dimples is varied from $0 \%$ (flat plane surface) to $20 \%$ for Reynolds numbers between 4,000 and 6,000. The various performance parameters are normalised by their respective values for the flat plane channel configuration, given by the subscript 0 . At $d / D=5 \%$, a small increase in $\mathrm{C}_{\mathrm{f}}$ is observed, and $\mathrm{C}_{\mathrm{f}}$ increases steadily as $d / D$ increases beyond $5 \%$. The Nusselt number however only shows a significant increase for $d / D>5 \%$, so that the volume goodness ratio shows a peak at $d / D=10 \%$. The results suggest that for efficient heat transfer enhancement, the dimples require a depth to diameter of at least $10 \%$. Thus, DES for the non-axisymmetric dimples have been carried out with $d / D=10 \%$ and $15 \%$ subsequently to study its heat transfer characteristics.

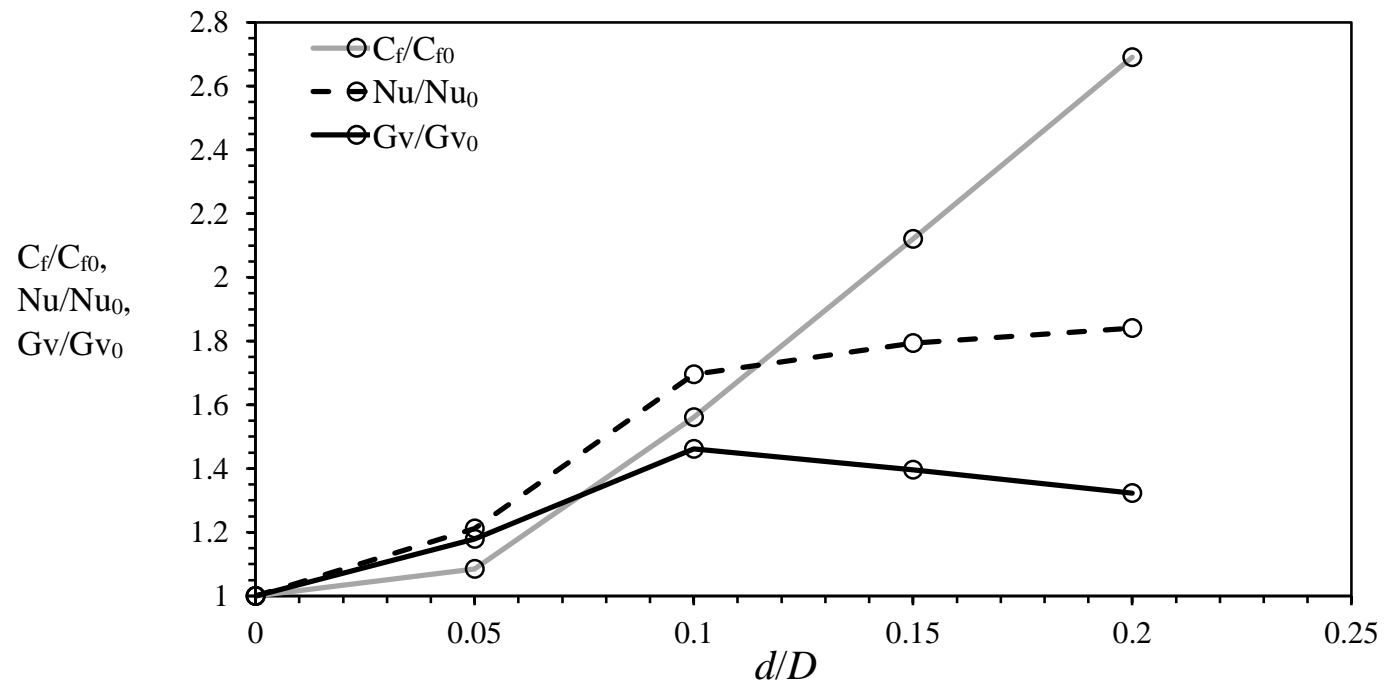

Fig. 2: Effect of changes in $d / D$ on axisymmetric dimple.

Changes in the streamwise movement of the deepest point from the dimple center $\Delta x / D$ shows significant effect on $C_{\mathrm{f}}$ and $\mathrm{Nu}$ as Fig. 3 shows. For both $d / D=10 \%$ and 15\%, the Nusselt number Nu increases steadily as $\Delta x / D$ increases. The trend for $\mathrm{C}_{\mathrm{f}}$ as $\Delta x / D$ increases however, is different for $d / D=10 \%$ and $15 \%$. With $d / D=15 \%$, the $\mathrm{C}_{\mathrm{f}}$ increases steadily as $\Delta x / D$ increases from $-7.5 \%$ to $15 \%$. The variation in $\mathrm{C}_{\mathrm{f}}$ for $d / D=10 \%$ however, shows a peak at $\Delta x / D=10 \%$. As $\Delta x / D$ increases from $15 \%$ to $17.5 \%$, the $\mathrm{C}_{\mathrm{f}}$ increases again. 


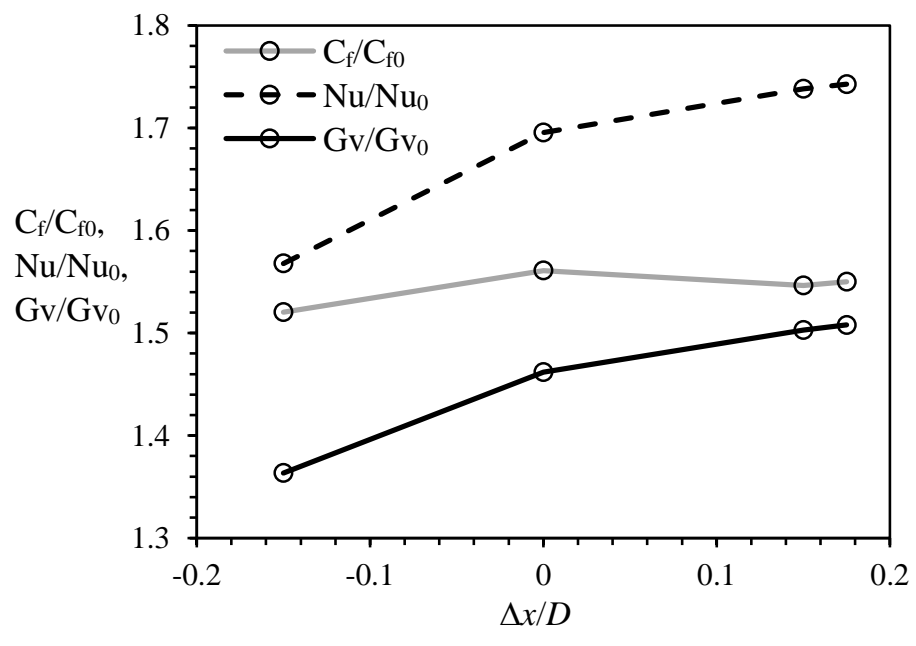

(a)

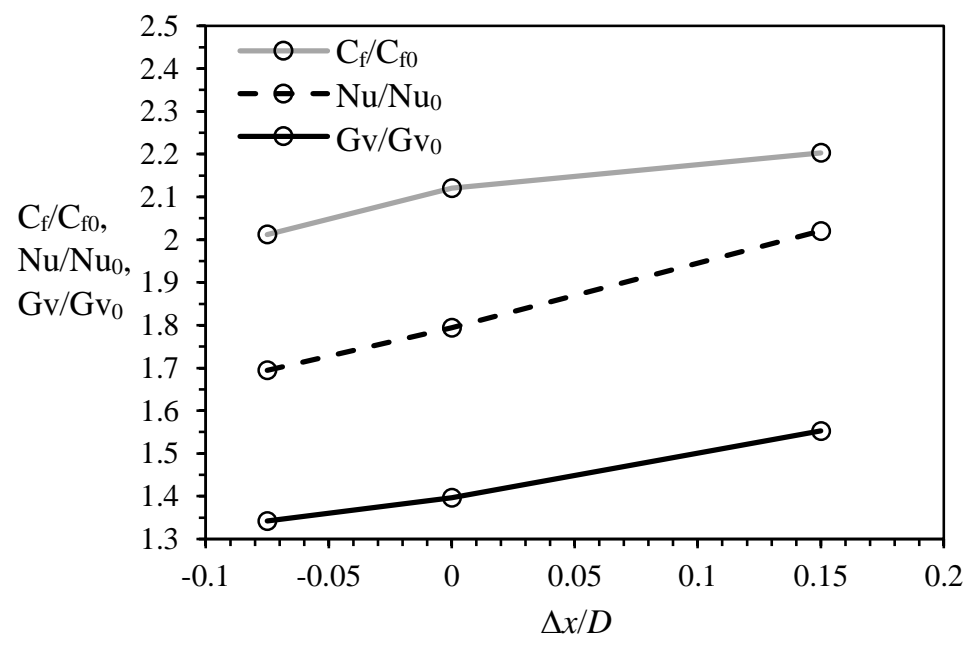

(b)

Fig. 3: Effect of changes in $\Delta x / D$ on dimple performance. (a) $d / D=10 \%$, (b) $d / D=15 \%$.

Similar to the axisymmetric dimples, the non-axisymmetric dimples with its deepest point moved streamwise show a similar variation in the dimple performance parameters as the dimple depth is varied as shown in Fig. 4 for a Reynolds number range between 4,000 and 6,000. It may be noteworthy that hence at $d / D=15 \%$, the movement of the deepest point downstream so that $\Delta x / D=15 \%$ results in a significant increase in $\mathrm{Nu}$, resulting in a similar increase in the goodness volume ratio $\mathrm{Gv}$. The figure shows that $\Delta x / D=15 \%, \mathrm{C}_{\mathrm{f}}$ when $d / D=10 \%$ is marginally lower than the axisymmetric dimple case, but when $d / D=15 \%, \mathrm{C}_{\mathrm{f}}$ is higher than the axisymmetric dimple case at the same dimple depth ratio $d / D$.

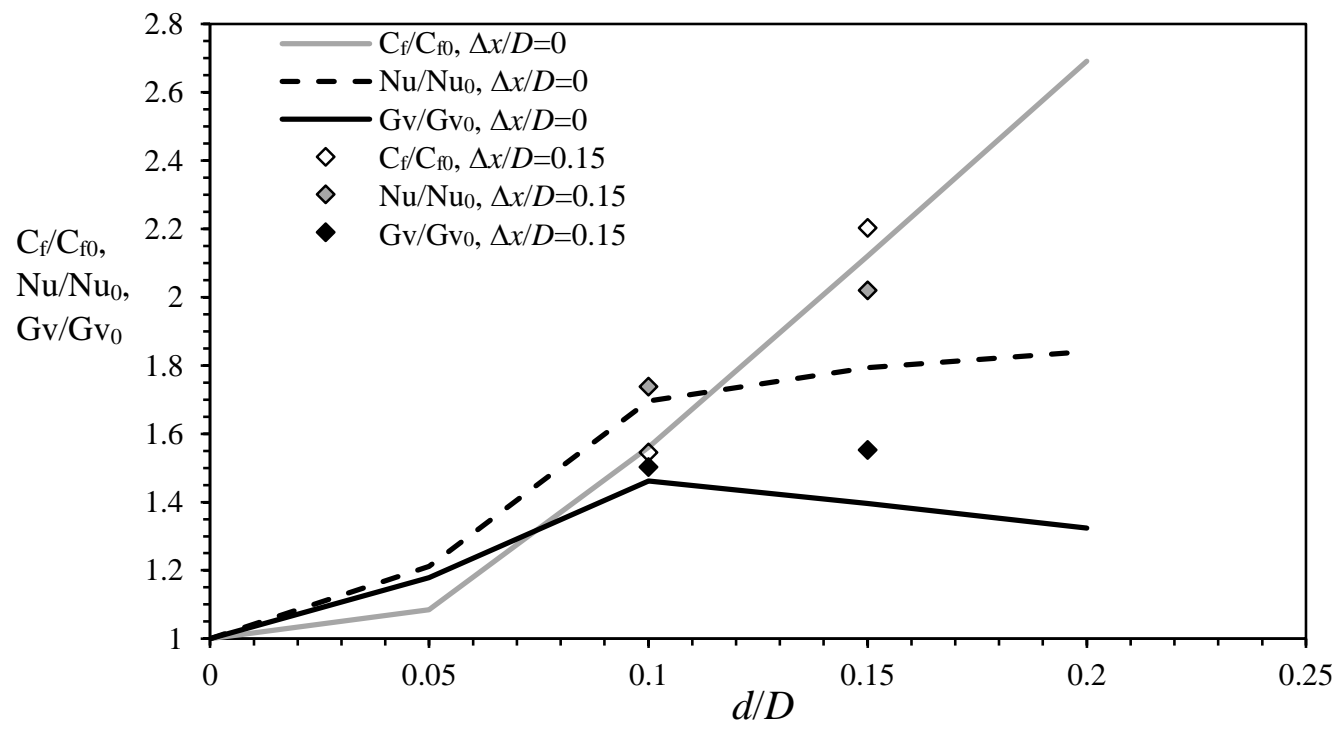

Fig. 4: Effect of changes in $d / D$ on dimple performance.

The DES shows that by moving the deepest point of the dimple downstream, the flow separation region that occurs at the upstream portion of the dimple is successfully reduced for both $d / D=10 \%$ and $d / D=15 \%$ as shown in Fig. 5. Fig. 5 (a) and (b) shows the streamlines for the dimple with $d / D=10 \%$ and they clearly show a reduction in the size of the flow separation region occurring at the upstream portion of the axisymmetric dimple when the deepest point is shifted downstream by $0.15 \mathrm{D}$. A similar change in the streamlines is also observed for the deeper dimple with $d / D=15 \%$, though not shown here. Fig. 5(c) and (d) shows contours of the vertical velocity v for the dimple with $d / D$ $=15 \%$, which also show the same reduction in flow separation region. Additionally, the contours also show the 
magnitude of the vertical component of the velocity. Fig. 5(d) shows the existence of a high velocity "ejection" region at the downstream edge of the dimple with the deepest point shifted downstream by $0.15 D$. The significant increase in the Nusselt number observed in Fig. 3 for both dimple depths when the deepest point is shifted downstream may be explained due to the following two reasons. The first reason is that as the deepest point is shifted downstream, the upstream flow separation region is reduced. The recirculating flow within the flow separation region of the dimple is not conducive for heat transfer and a reduction in this recirculating flow results in an increase in the heat transfer capability of the dimple. The second reason is that the existence of an ejection region consisting of fast vertically moving fluid at the downstream, edge of the dimple increases flow mixing and increases the heat transfer of the dimple at the downstream edge as well. These two reason explain the significant increase in the Nusselt number as the deepest point of the dimple is shifted downstream. The opposite is also true and a reduction in $\mathrm{Nu}$ is observed if the deepest point of the dimple is shifted upstream instead of downstream as Fig. 3 also shows.

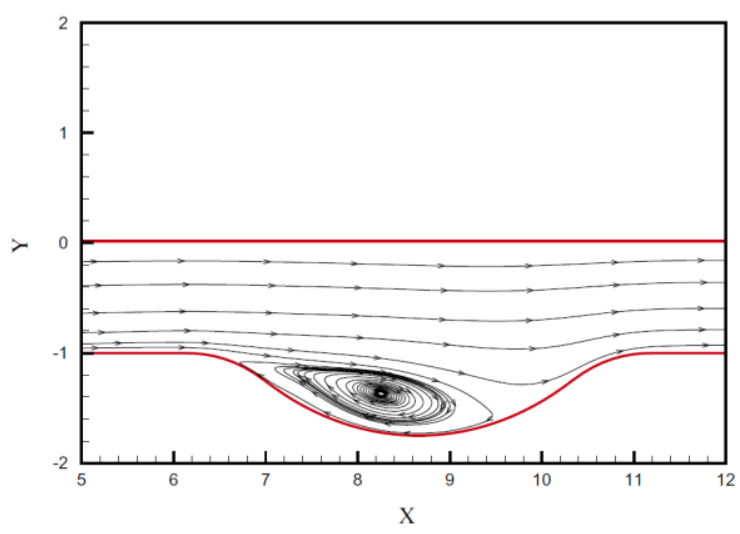

(a) $d / D=10 \%, \Delta x / D=0 \%$.

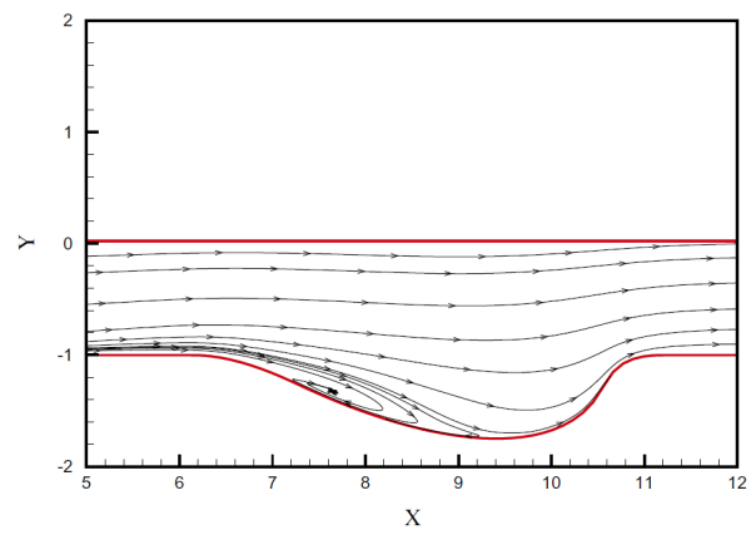

(b) $d / D=10 \%, \Delta x / D=15 \%$.

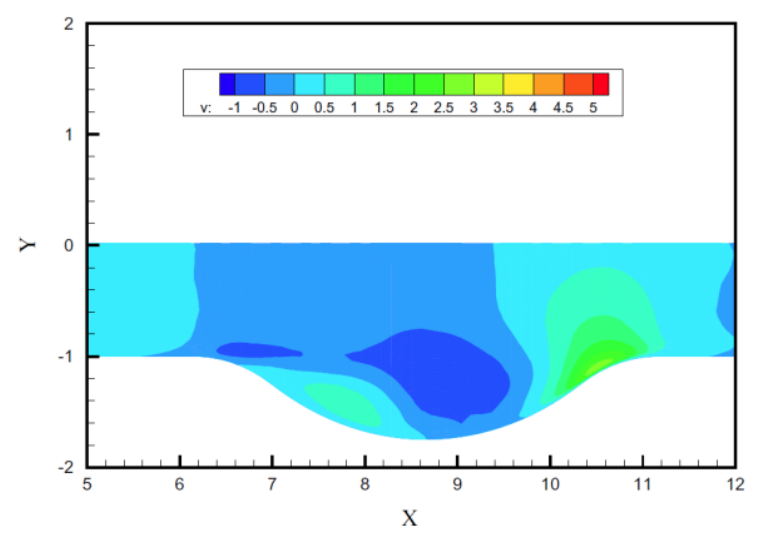

(c) $d / D=15 \%, \Delta x / D=0 \%$.

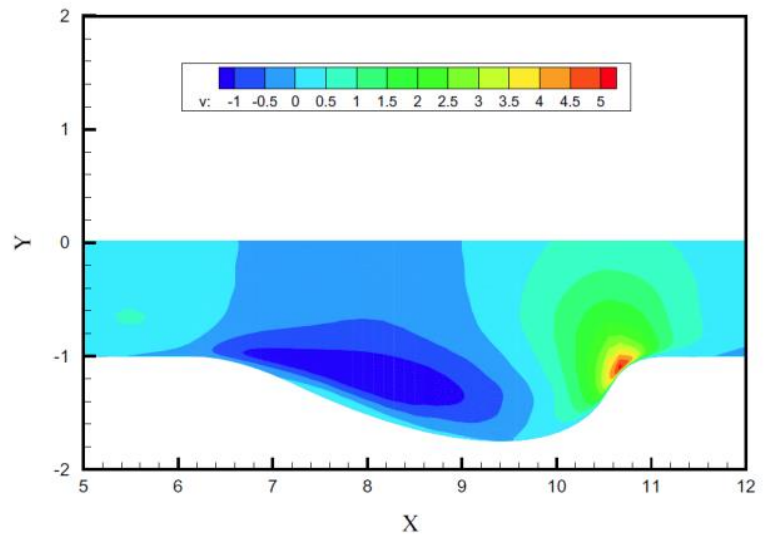

(d) $d / D=15 \%, \Delta x / D=15 \%$.

Fig. 5: Reduction in flow separation region at $\Delta x / D=15 \%$ compared to axisymmetric dimple with $\Delta x / D=0 \%$.

The results from complimentary experiments conducted with shallow dimples with $d / D=5 \%$ also agree with these findings [12]. Details about the experiment are given in [10,17], and the results show that moving the deepest point downstream from the dimple center generally reduces the pressure loss while moving it forward increases the pressure loss due to the dimples. The experiments are conducted at a higher Reynolds number range compared to the DES, from 13000 to 55000 to avoid transition instabilities occurring at lower Reynolds numbers. The variation in the friction factor compared to the plane channel case for the experiments is shown in Fig. 6 for a Reynolds number of 13,000. Its shows a steady reduction in $\mathrm{C}_{\mathrm{f}} / \mathrm{C}_{\mathrm{f} 0}$ as $\Delta x / D$ increases from $-20 \%$ to $10 \%$. As $\Delta x / D$ increases above $10 \%, \mathrm{C}_{\mathrm{f}} / \mathrm{C}_{\mathrm{f} 0}$ increases again. A minimum value of $\mathrm{C}_{\mathrm{f}} / \mathrm{C}_{\mathrm{f} 0}$ is observed at $\Delta x / D=10 \%$. Though the results for $\mathrm{C}_{\mathrm{f}} / \mathrm{C}_{\mathrm{f} 0}$ in Fig. 6 are for a Reynolds number of 13,000, which is higher than the DES carried out at a Reynolds number of 4000 to 6000, the authors believe that 
comparisons of the trend among them remains valid since $\mathrm{C}_{\mathrm{f}} / \mathrm{C}_{\mathrm{f} 0}$ is relatively insensitive to such small changes in Reynolds number compared to the effects of parameters such as $d / D$ and $\Delta x / D$.

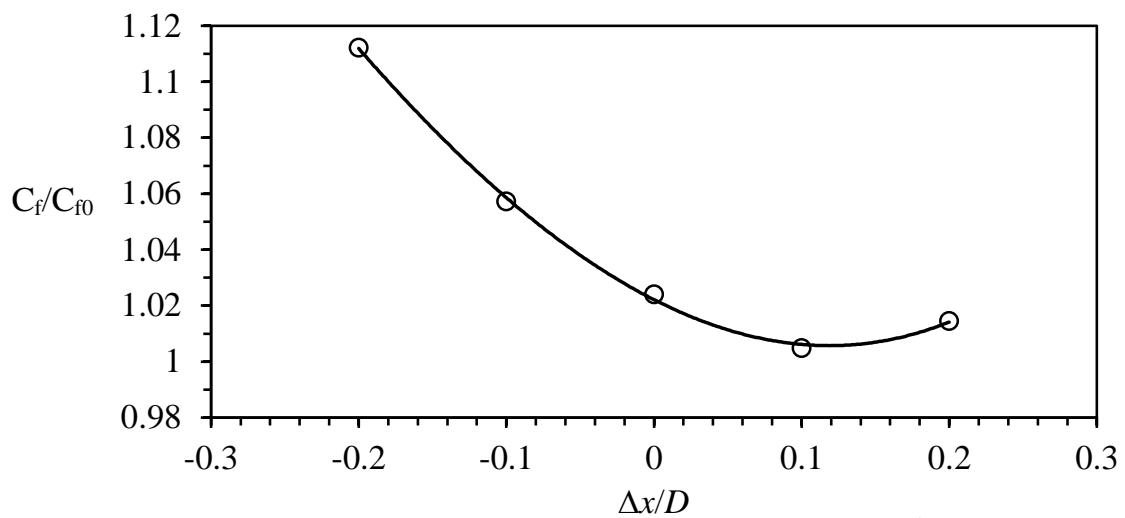

Fig. 6: Variation of $\mathrm{C}_{\mathrm{f}}$ with $\Delta x / D$ for dimples with $d / D=5 \%$.

As $\Delta x / D$ increases from zero, the axisymmetric case, the upstream wall slope is reduced, but at the expense of the downstream wall slope. The upstream wall slope reduces as the deepest point is shifted downstream, but the downstream wall slope increases. The latter results in significant flow impingement at the downstream edge. This is observed in the streamlines and velocity contours in Fig. 5(b) and (d). Significant flow impingement can result in increased $\mathrm{C}_{\mathrm{f}} / \mathrm{C}_{\mathrm{f} 0}$ and pressure losses. For shallow dimples with low $d / D$ and at low values of $\Delta x / D$, the downstream wall slope though increased, remains relatively low so that flow impingement is not particularly severe and increase in $\mathrm{C}_{\mathrm{f}} / \mathrm{C}_{\mathrm{fo}}$ and pressure loss that results from the impingement is not significant. The reduction in flow separation and flow recirculation at the upstream portion of the dimple results in a net reduction in $\mathrm{C}_{\mathrm{f}} / \mathrm{C}_{\mathrm{f} 0}$ and increase in the Nusselt number, improving dimple performance. However, with increasing dimple depth to diameter ratio $d / D$ and higher values of $\Delta x / D$, the downstream wall is increased significantly, resulting in significant flow impingement at the downstream edge of the dimple, hence leading to increased skin friction and pressure drag at this portion of the dimple. The increase in skin friction and pressure drag arising from the flow impingement can overcome the reduction in pressure drag due to the reduction in flow separation in the upstream portion of the dimple, resulting in a net increase in $\mathrm{C}_{\mathrm{f}} / \mathrm{C}_{\mathrm{f} 0}$ or pressure loss. Hence for the deeper dimple with $d / D=15 \%$, increasing $\Delta x / D$ leads to a steady increase in $\mathrm{C}_{\mathrm{f}} / \mathrm{C}_{\mathrm{f}}$ as Fig. 3 shows.

Comparison between Fig. 3 and Fig. 6 shows a significant change in the trend in $\mathrm{C}_{\mathrm{f}}$ as the streamwise movement of the deepest point $\Delta x / D$ is varied for dimples with different $d / D$. For dimples with $d / D=15 \%$, a steady increase in $C_{\mathrm{f}}$ is observed as $\Delta x / D$ increases. No peaks or trough is observed for $\mathrm{C}_{\mathrm{f}} / \mathrm{C}_{\mathrm{f} 0}$ as $\Delta x / D$ increases from $-7.5 \%$ to $0 \%$ to $15 \%$. For dimples with $d / D=10 \%$, a local peak is observed for $\mathrm{C}_{\mathrm{f}} / \mathrm{C}_{\mathrm{fo}}$ at $\Delta x / D=10 \%$ as $\Delta x / D$ increases from $-15 \%$ to $0 \%$ to $15 \%$. For dimples with $d / D=5 \%$, Fig. 6 shows a local minimum in the value of $\mathrm{C}_{\mathrm{f}} / \mathrm{C}_{\mathrm{f} 0}$ at $\Delta x / D=10 \%$ for such shallow dimples. However, for a more valid comparison with Fig. 3, only the same values of $\mathrm{C}_{\mathrm{f}} / \mathrm{C}_{\mathrm{f} 0}$ as Fig. 3 should be noted for the comparison. On visually studying Fig. 6 , one would conclude that for dimples with $d / D=5 \%, \mathrm{C}_{\mathrm{f}} / \mathrm{C}_{\mathrm{f} 0}$ decreases steadily as $\Delta x / D$ increases through the three values of $-15 \%$ to $0 \%$ to $15 \%$. There is thus a change in the trend from a steady reduction in $\mathrm{C}_{\mathrm{f}} / \mathrm{C}_{\mathrm{f} 0}$ as $\Delta x / D$ increases for dimples with $d / D=5 \%$, to a local maximum for $\mathrm{C}_{\mathrm{f}} / \mathrm{C}_{\mathrm{f} 0}$ appearing at $\Delta x / D=10 \%$ for dimples with $d / D=10 \%$, to a steady increase in $\mathrm{C}_{\mathrm{f}} / \mathrm{C}_{\mathrm{f} 0}$ as $\Delta x / D$ increases for dimples with $d / D=15 \%$. It should be noted that this trend is only apparent at lower values of $\Delta x / D$. When $\Delta x / D$ is greater than $<15 \%$, the downstream wall is steep enough to result in significant flow impingement at the downstream edge of the dimple and $\mathrm{C}_{\mathrm{f}} / \mathrm{C}_{\mathrm{f}}$ increases with further increases in $\Delta x / D$ regardless of dimple depth $d / D$.

The reason for this is likely due to the non-linear behaviour of the flow and the different rates at which the two effects of flow separation and flow impingement vary with changes in the wall slope steepness. As the dimple depth to diameter $d / D$ increases, the average wall slope of the upstream and downstream portion increases. Dimples with large $d / D$ have relatively steep upstream and downstream wall slopes. A shift in the deepest point upstream increases the upstream wall slope, but reduces the downstream wall slope. Because the initial wall slope was already very steep and 
flow separation was already significant, a small increase in wall slope further does not cause a significant further increase in the flow separation region. However, a reduction in the steep downstream wall results in a comparatively significant reduction in flow impingement of the downstream wall. The result is that for deeper dimples, $\mathrm{C}_{\mathrm{f}} / \mathrm{C}_{\mathrm{f} f}$ reduces as $\Delta x / D$ decreases, as is the case observed for $d / D=15 \%$ (Fig. 3). For shallow dimples, both upstream and downstream walls are relatively shallow. The flow separation region upstream is relatively small and flow impingement at the downstream edge is relatively mild. As the deepest point is shifted upstream and $\Delta x / D$ decreases, the flow separation region is increased significantly, while the already mild flow impingement at the downstream end is only slightly reduced. The result is that for shallow dimples, $\mathrm{C}_{\mathrm{f}} / \mathrm{C}_{\mathrm{f} 0}$ increases due to the significant increase in flow separation as $\Delta x / D$ decreases. Between these two trends for deeper and shallower dimples, when $d / D=10 \%$, an intermediate exist where both the flow separation at the upstream portion and flow impingement at the downstream edge is equally significant. Movement of the deepest point slightly upstream of the dimple center results in a significant reduction in flow impingement with a negligible increase in flow separation. A movement of the deepest point slightly downstream of the dimple center results in a significant reduction in flow separation with a negligible increase in flow impingement. The result is that at when $d / D=10 \%$, a peak in $\mathrm{C}_{\mathrm{f}} / \mathrm{C}_{\mathrm{f} 0}$ is observed at $\Delta x / D=0$, when the dimple is axisymmetric.

\section{Conclusion}

Numerical simulations and experiments were conducted on circular axisymmetric as well as non-axisymmetric dimples with their deepest point shifted in a streamwise direction as methods to reduce the flow separation region commonly observed at the upstream portion of dimples. The aim was to reduce pressure drag due to the flow separation and raise the heat transfer efficiency of dimple surfaces. The results show that though shallow dimples with depth to diameter ratios of $5 \%$ have relatively lower friction factors due to reduced flow separation, their heat transfer enhancement ability is however limited. Deeper dimples with depth to diameter ratios of at least $10 \%$ show significantly higher heat transfer compared to shallow dimples and are more favourable as candidates for heat transfer applications despite higher flow separation and recirculation observed with these deeper dimples.

Improvements of the heat transfer efficiency can be made by shifting the deepest point of the circular dimple streamwise. Shifting the deepest point downstream reduces the wall slope of the uptream edge and has the effect of reducing the flow separation and recirculation region commonly observed at the upstream portion of the dimples. The shift in the deepest point downstream also has the effect of increasing the wall slope of the downstream dimple edge, which leads to higher flow impingement at the downstream edge as well as higher fluid ejection out of the dimple in this region. Both the reduction in flow recirculation and increased fluid ejection increases the heat transfer ability of the dimple. Its effect on the heat transfer efficiency however, depends not only on the change in the heat transfer ability, but also the accompanying change in the friction factor.

The dimples exhibit various trends in the friction factor depending on the dimple depth to diameter ratio. Shallow dimples with depth to diameter ratios of 5\% show decreasing friction factors as the deepest point is shifted downstream due to the more significant effect that the reduction in the flow separation region has on the friction factor for shallow dimples. Deeper dimples with depth to diameter ratios of $15 \%$ show the opposite trend, and the friction factor increases as the deepest point is shifted downstream. This is likely due to the relatively steeper walls of the dimple resulting in the increase in flow impingement at the downstream wall having a greater effect on the friction factor. In all cases however, movement of the deepest point beyond about $15 \%$ of the diameter from the dimple center results in increasing friction factor as the deepest point is further shifted downstream. This is due to the increasing downstream wall slope that results, resulting in significant flow impingement and increasing the pressure drag and consequently, the friction factor.

Further work is required to further optimize other parameters of the asymmetric dimple, such as channel height, spacing of dimples, print diameter and dimple edge radius.

\section{References}

[1] Y. Chen, Y. T. Chew, and B. C. Khoo, "Enhancement of heat transfer in turbulent channel flow over dimpled surface," Int. J. Heat and Mass Transfer, vol. 55, pp. 8100-8121, 2012.

[2] Y. Chen, Y. T. Chew, and B. C. Khoo, "Heat transfer and flow structure in turbulent channel flow over protrusions," Int. J. Heat and Mass Transfer, vol. 66, pp. 177-191, 2013. 
[3] Y. Chen, Y. T. Chew, and B. C. Khoo, "Heat transfer and flow structure on periodically dimple-protrusion patterned walls in turbulent channel flow," Int. J. Heat and Mass Transfer, vol. 78, pp. 871-882, 2014

[4] P. Ligrani, J. Harrison, G. Mahmmod and M. Hill, "Flow structure due to dimple depressions on a channel surface," Phys. Fluids, vol. 13, pp. 3442-3451, 2001.

[5] S. Isaev, N. Kornev, A. Leontiev and E. Hassel, "Influence of the Reynolds number and the spherical dimple depth on turbulent heat transfer and hydraulic loss in a narrow channel," Int. J. Heat and Mass Transfer, vol. 53, no. 1, pp. 178-197, 2010.

[6] J. Turnow, N. Kornev and S. E. H. Isaev, "Vortex mechanism of heat transfer enhancement in a channel with spherical and oval dimples," Heat and Mass Transfer, vol. 47, pp. 301-311, 2011.

[7] M. Chyu, Y. Yu, H. Ding, J. Downs and F. Soechting, "Concavity enhanced heat transfer in an internal cooling passage," in: ASME, Paper No. 97-GT-437, 1997.

[8] J. Doo, H. Yoon and M. Ha, "Study on improvement of compactness of a plate heat exchanger using a newly designed primary surface," Int. J. Heat and Mass Transfer, vol. 53, pp. 5733-5746, 2010.

[9] S. Isaev, A. Leont'ev, A. Mityakov, I. Pyshnyi and I., Usachov, "Intensification of tornado turbulent heat exchange in asymmetric holes on a plane wall," J. Eng. Physics Thermophysics, vol. 76, no. 2, pp. 266-270, 2003.

[10] C. M. Tay, B. C. Khoo and Y. T. Chew, "Mechanics of drag reduction by shallow dimples in channel flow," Phys. Fluids, vol. 27, no. 3, pp. 035109, 2015.

[11] C. M. Tay, Y. T. Chew, B. C. Khoo and J. B. Zhao, "Development of flow structures over dimples," Exp. Thermal Fluid Sci., vol. 52, pp. 278-287, 2014.

[12] C. M. Tay, and T. T. Lim, "Drag reduction with non-axisymmetric dimples," 35th AIAA Applied Aerodynamics Conference, AIAA paper 2017-3569, 2017.

[13] P. R. Spalart, W. H. Jou, M. Strelets and S. R. Allmaras, "Comments on the feasibility of LES for wings, and on a hybrid RANS/LES approach," 1st AFOSR Int. Conf. on DNS/LES, Greyden Press, Columbus, OH, 1997.

[14] M. Shur, P. R. Spalart, M. Strelets and A. Travin, "Detached-Eddy Simulation of an Airfoil at High Angle of Attack," Eng Turb Modelling and Exp. vol. 4, pp. 669-678, 1999.

[15] N. V. Nikitin, F. Nicoud, B. Wasistho, K. D. Squires and P. R. Spalart, "An approach to wall modeling in large-eddy simulations," Phys Fluids, vol. 12, pp. 1629-1632, 2000.

[16] R. Shah and A. London, "Laminar flow forced convection in ducts: a source book for compact heat exchanger analytical data," Academic Press, New York, NY, 1978.

[17] C. M. Tay, "Determining the effect of dimples on drag in a turbulent channel flow," 49th AIAA Aerospace Sciences Meeting and Exhibit, AIAA Paper 2011-0682, 2011. 